\title{
The use of Human Inflammatory Response and Autoimmunity RT2 lncRNA PCR Array for plasma examination in breast cancer patients prior to therapy
}

\author{
M. STERBOVA ${ }^{1}$, E. PAZOURKOVA ${ }^{1}$, S. SANTOROVA ${ }^{1}$, I. ZEDNIKOVA ${ }^{1}$, P. TESAROVA ${ }^{2}$, M. KORABECNA ${ }^{1, *}$ \\ ${ }^{1}$ Institute of Biology and Medical Genetics, First Faculty of Medicine, Charles University and General University Hospital in Prague, Prague, \\ Czech Republic; ${ }^{2}$ Department of Oncology, First Faculty of Medicine, Charles University and General University Hospital in Prague, Prague, \\ Czech Republic
}

${ }^{*}$ Correspondence: marie.korabecna@lf1.cuni.cz

Received September 7, 2018 / Accepted February 20, 2019

\begin{abstract}
Long non-coding RNAs (lncRNAs) are defined as RNA molecules longer than 200 nucleotides with poor protein-coding capacity and key functions in regulation of gene expression. Dysregulations of lncRNAs (e.g. HOTAIR and MALAT I) were detected in plasma of breast cancer (BC) patients. Plasma samples are examined as liquid biopsies for purposes of non-invasive diagnostics therefore the research of plasma lncRNAs as potential plasma biomarkers became highly topical. 84 lncRNAs were profiled in 18 plasma samples - 9 BC patients and 9 age-matched healthy - using Human Inflammatory Response \& Autoimmunity RT2 lncRNA PCR Array. Total RNA from plasma samples was isolated using miRNeasy Serum/ Plasma Kit. Although a pre-amplification recommended for quantification from small starting RNA amounts was used, only 3 lncRNAs (A2ML1-AS1, GAS5 and SNHG5) were detected in all plasma samples. A total of 72 lncRNAs (e.g. HOTAIR or MALAT I) were detected only in some samples and 9 lncRNAs were not detected in any samples. No significant differences were observed in levels of plasma lncRNAs between the BC patients and healthy controls despite the fact that our panel contained also the lncRNAs whose levels were previously reported as significantly different in plasma or cancer tissues (e.g. GAS5, HOTAIR, MALAT I) in BC patients. Detection of lncRNAs in plasma is due to their low concentrations quite difficult as compared with tissues. Our findings suggest that analysis of plasma lncRNAs using this technology is not suitable for use as non-invasive diagnostic tool in BC patients.
\end{abstract}

Key words: breast cancer, long non-coding RNA, plasma, HOTAIR, MALAT1, biomarkers

Final diagnosis of breast cancer (BC) is still dependent on histological examination because the blood markers have limited efficacy [1]. Therefore, the search for more effective non-invasive markers for early BC detection is highly topical. In recent years, plasma samples have been examined as liquid biopsies with regards to their content of tumor derived nucleic acids [2-5]. It has been shown that long non-coding RNAs (lncRNAs) which are released from tumor tissues, can be also detected in the circulation $[6,7]$. LncRNAs are usually considered as a heterogeneous group of RNAs. They are defined as RNA molecules longer than 200 nucleotides with poor protein-coding capacity [8]. LncRNAs serve as regulators controlling the expression of genes at epigenetic, transcriptional and post-transcriptional levels. LncRNAs regulate various biological processes such as stability of telomeres, chromatin modification, $\mathrm{X}$ chromosome inactivation, gene imprinting, transcription activation and inhibition and miRNA activity [9-13].
Dysregulation of lncRNAs is associated with a large spectrum of human diseases, including different types of cancer $[6,7,14]$. Therefore, their research and potential use as biomarkers became the focus of interest. Dysregulations of lncRNAs in tissues were detected also in BC $[15,16]$.

Recently, Yu G. et al. [17] performed a meta-analysis to evaluate the results of studies dealing with testing lncRNAs as biomarkers for early BC detection. 484 studies were found but only ten of them passed the stringent exclusion criteria. The Quality Assessment of Diagnosis Accuracy Studies II criteria (QUADAS) [18] were used to select the eligible studies. The selected studies comprised 835 BC patients and 725 paired controls. Five selected studies examined plasma samples, four studies dealt with serum and one study analyzed urine of BC patients. The meta-analysis resulted in conclusion that lncRNAs HOTAIR, MALAT I and H19 may serve as useful biomarkers for non-invasive early $\mathrm{BC}$ detection. The best specificity (0.89) and AUC (0.86) were 
achieved with lncRNA HOTAIR which was superior to lncRNAs MALAT I and H19, but the best sensitivity (0.83) was reported for MALAT I. Serum-based analysis provided better sensitivity (0.83) than plasma-based analysis, whereas the plasma-based examination led to better specificity $(0.88)$ [17]. All patients in analyzed cohorts were selected from Chinese population - unfortunately there are no similar data for other populations.

HOX antisense intergenic RNA (HOTAIR) causes gene silencing through its interactions with epigenetic regulators such as histone methyltransferase PRC2 (polycomb repressive complex 2) and histone demethylase LSD1 (lysine specific demethylase 1A). HOTAIR serves as a scaffold recruiting these complexes to the target gene loci. There is a competition between HOTAIR and BRCA1 for binding to PRC2. HOTAIR promotor contains also multiple estrogen response elements, therefore HOTAIR expression may be activated by estradiol in estrogen receptor positive breast cancer cells [19].

MALAT I (Metastasis-associated lung adenocarcinoma transcript-1) regulates gene expression and it may modulate also alternative splicing. Its elevated expression was detected in primary breast tumors and its mutant forms were found in luminal breast cancer [20]. The fact that activities of immune system are crucial for elimination of cancer cells and developing tumors is well known. The roles of lncRNAs in these processes are described and further studied [21,22].

Due to all above-mentioned facts, we managed this pilot study to test the performance of the Human Inflammatory Response and Autoimmunity $\mathrm{RT}^{2}$ lncRNA PCR Array (QIAGEN, Germany):

$1 /$ for the selection of new potential biomarkers associated with immune response against cancer cells among 84 lncRNAs involved in immune response regulation in plasma samples from randomly selected patients with histologically confirmed $\mathrm{BC}$ diagnosis prior to the start of therapy in comparison with age-matched healthy females

2/ for comparison of lncRNAs HOTAIR and MALAT I levels included in the array to evaluate their potential for non-invasive breast cancer diagnosis.

\section{Patients and methods}

Plasma samples were obtained from $9 \mathrm{BC}$ patients and 9 age-matched healthy women. The average age in healthy control group was $39.5 \pm 12.4$ years; the average age of breast cancer patients was $39.7 \pm 11.9$ years. The differences between the ages of females involved in these groups were evaluated as statistically non-significant (Mann Whitney test). The diagnosis of $\mathrm{BC}$ was histologically confirmed in all patients (Table 1). Plasma samples for analysis were obtained prior to the start of therapy. The age-matched healthy volunteers were not exposed to any disease or injury. All participants of the study signed the informed consent. The study has been carried out in accordance with Declaration of Helsinki for experiments involving humans. The study was approved by the Ethics committee of the 1st Faculty of Medicine of Charles University and General Faculty Hospital in Prague.

LncRNA PCR array analysis. The total cell-free RNA from $200 \mu$ of plasma was isolated using miRNeasy Serum/ Plasma Kit (QIAGEN, Germany) and finally eluted into $14 \mu \mathrm{l}$ of RNase-free water. We detected very low RNA concentrations $(0.1 \mathrm{ng} / \mu \mathrm{l}$ in average) using Bioanalyzer 2100 (Agilent) and Small RNA kit (Agilent), which is focused on quantification of small RNA species in a total RNA preparation. When we measured the total RNA concentrations on NanoDrop 8000 (Thermo Fischer Scientific), we obtained higher values (12 $\mathrm{ng} / \mu \mathrm{l}$ in average). As the RIN values are based on $18 \mathrm{~S}$ and 28S RNA peaks and the presence of ribosomal RNAs in bodily fluids is indicative of contamination by cells or cell debris, the ribosomal bands are usually very low or absent and therefore not suitable as integrity control for RNA isolated from plasma samples. Additionally, the short fragments of RNA ( $<1000 \mathrm{nt})$ may be perceived by the Bioanalyzer as degraded RNA [23]. According to these facts, the average RIN value in our RNA samples was 2 . Due to very low concentrations of total RNA in the obtained samples, $8 \mu$ of each sample was transcribed into cDNA (complementary DNA) and then pre-amplified using $\mathrm{RT}^{2}$ PreAMP cDNA Synthesis Kit (QIAGEN, Germany) with specific $\mathrm{RT}^{2}$ lncRNA PreAMP Primer Mix (QIAGEN, Germany) according to the manufacturer's protocol. $102 \mu \mathrm{l}$

Table 1. BC patients - tumor characteristics.

\begin{tabular}{lccccccccc}
\hline \multirow{2}{*}{ Characteristic } & \multicolumn{10}{c}{ BC patient no. } \\
\cline { 2 - 8 } & $\mathbf{1}$ & $\mathbf{2}$ & $\mathbf{3}$ & $\mathbf{4}$ & $\mathbf{5}$ & $\mathbf{6}$ & $\mathbf{7}$ & $\mathbf{8}$ & $\mathbf{9}$ \\
\hline BC type & NST & NST & NST & NST & NST & NST & LC & NST & NST \\
ER positivity (\%) & $100 \%$ & 0 & $65 \%$ & 0 & 0 & $20 \%$ & $95 \%$ & $80 \%$ & $100 \%$ \\
PR positivity (\%) & $70 \%$ & 0 & $40 \%$ & 0 & 0 & $20 \%$ & $95 \%$ & $60 \%$ & $100 \%$ \\
HER2 positivity & + & - & + & - & - & - & - & + & + \\
KI 67 positivity (\%) & $30 \%$ & $90 \%$ & $40 \%$ & $90 \%$ & $70 \%$ & $90 \%$ & $15 \%$ & $70 \%$ & $80 \%$ \\
Grading & G2 & G3 & G3 & G3 & G3 & G3 & G2 & G3 & G3 \\
\hline
\end{tabular}

NST = Invasive carcinoma of no special type, LC = lobular carcinoma; ER = estrogen receptor, $\mathrm{PR}=$ progesterone receptor, HER $2=$ human epidermal growth factor receptor, KI 67 = nuclear antigen encoded by $M K I 67$ gene 
of pre-amplified sample was added to $1275 \mu \mathrm{l}$ of $\mathrm{RT}^{2}$ SYBR Green ROX qPCR Mastermix (Qiagen, Germany) and $1173 \mu \mathrm{l}$ RNase-free water and then each sample was loaded on to a commercially supplied Human Inflammatory Response \& Autoimmunity $\mathrm{RT}^{2}$ lncRNA PCR Array format A (QIAGEN, Germany) which comprise a gene-specific assays for 84 lncRNAs validated or predicted to regulate the expression of pro-inflammatory and anti-inflammatory genes and microRNAs and five reference genes. Amplification was run on an ABI 7900HT Fast Real-Time PCR System (Applied Biosystems, USA). Normalization to reference genes ACTB and RPLP0 as well as normalization to global mean were used. Both methods of normalization led to the same result.

Statistical analysis. The obtained data were compared using Mann-Whitney tests with Benjamini-Hochberg correction to multiple testing. For selection of potentially differentially expressed lncRNA the following criteria were used: fold change $>2$ and $\mathrm{p}<0.05$ (after Benjamini-Hochberg correction). The statistical analyses were performed using Expression Suite software (Life Technologies, New York, USA), qBase+ (Biogazelle NV, Belgium) and STATISTICA version 10 (StatSoft, Inc., Tulsa, USA).

\section{Results}

Although the pre-amplification step recommended for quantification from small starting RNA amounts was used, only 3 lncRNAs (A2ML1-AS1, GAS5 and SNHG5) were detected in all plasma samples $(\mathrm{Cq}<35)$. A total of 72 lncRNAs (e.g. HOTAIR) were detected only in some samples and 9 lncRNAs were not detected in any samples $(\mathrm{Cq}$ undetermined) (Figure 1).

No significant differences were observed in levels of plasma lncRNAs between the BC patients and healthy controls despite the fact that our panel contained the lncRNAs whose expressions were previously reported as significantly different at the level of cancer and control tissues (e.g. GAS5, HOTAIR, MALAT I) [15-17] (Figures 2 and 3). The levels of the two most abundant lncRNAs A2ML1-AS1 and SNHG5 - which are associated with immune system functions and which were detected in all examined samples - also did not differ significantly between patients and age-matched controls (Figure 3).

\section{Discussion}

In our pilot study, we detected low plasma levels of lncRNAs associated with functions of immune system despite the fact that exosomes are known transporters of lncRNA [24]. Detection of lncRNAs in plasma seems to be more difficult when compared with tissues [16, 25]. Using the identical technological platform as Schlosser et al. [25], we obtained very similar results as were reported by this group: Despite the pre-amplification step, the majority of lncRNAs was undetectable or only sporadically detectable

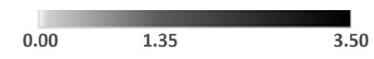

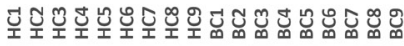

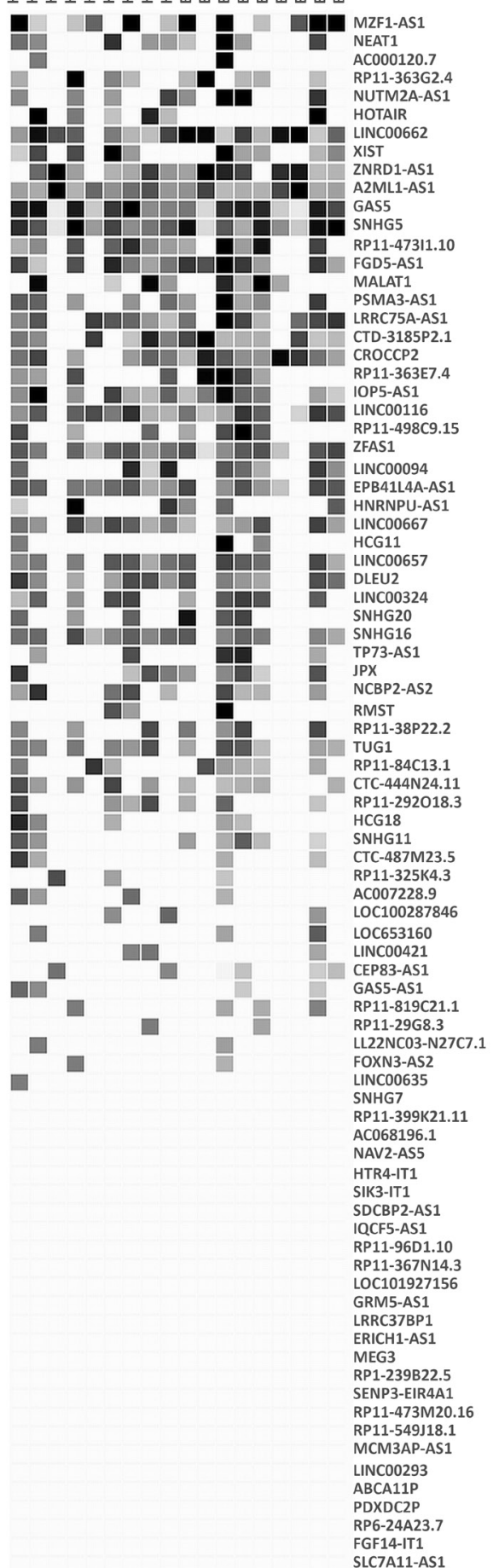

Figure 1. Relative quantification of lncRNAs in plasma of $\mathrm{BC}$ patients and healthy controls $(\mathrm{Cq}<35$, normalization by global mean). The columns represent healthy controls (HC) and $\mathrm{BC}$ patients $(\mathrm{BC})$ and the rows represent different lncRNAs. White color corresponds with fold change $=0.00(\mathrm{Cq} \geq 35$ and $\mathrm{Cq}$ undetermined), black color corresponds with fold change $\geq 3.50$. The heatmap was created in the program morpheus (https://software.broadinstitute.org/morpheus/) using the tool Nearest Neighbors and metric Euclidean distance. 


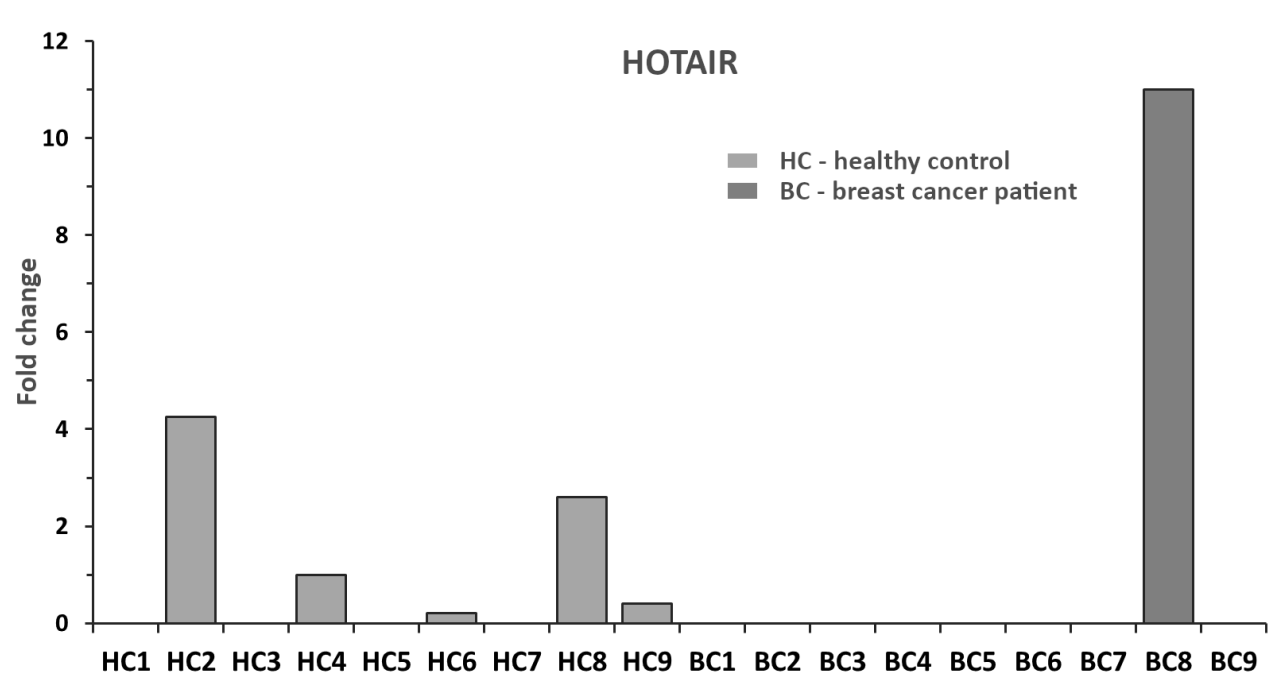

Figure 2. Relative quantification of HOTAIR in individual plasma samples of $\mathrm{BC}$ patients and healthy controls (Cq<35, normalization by global mean).
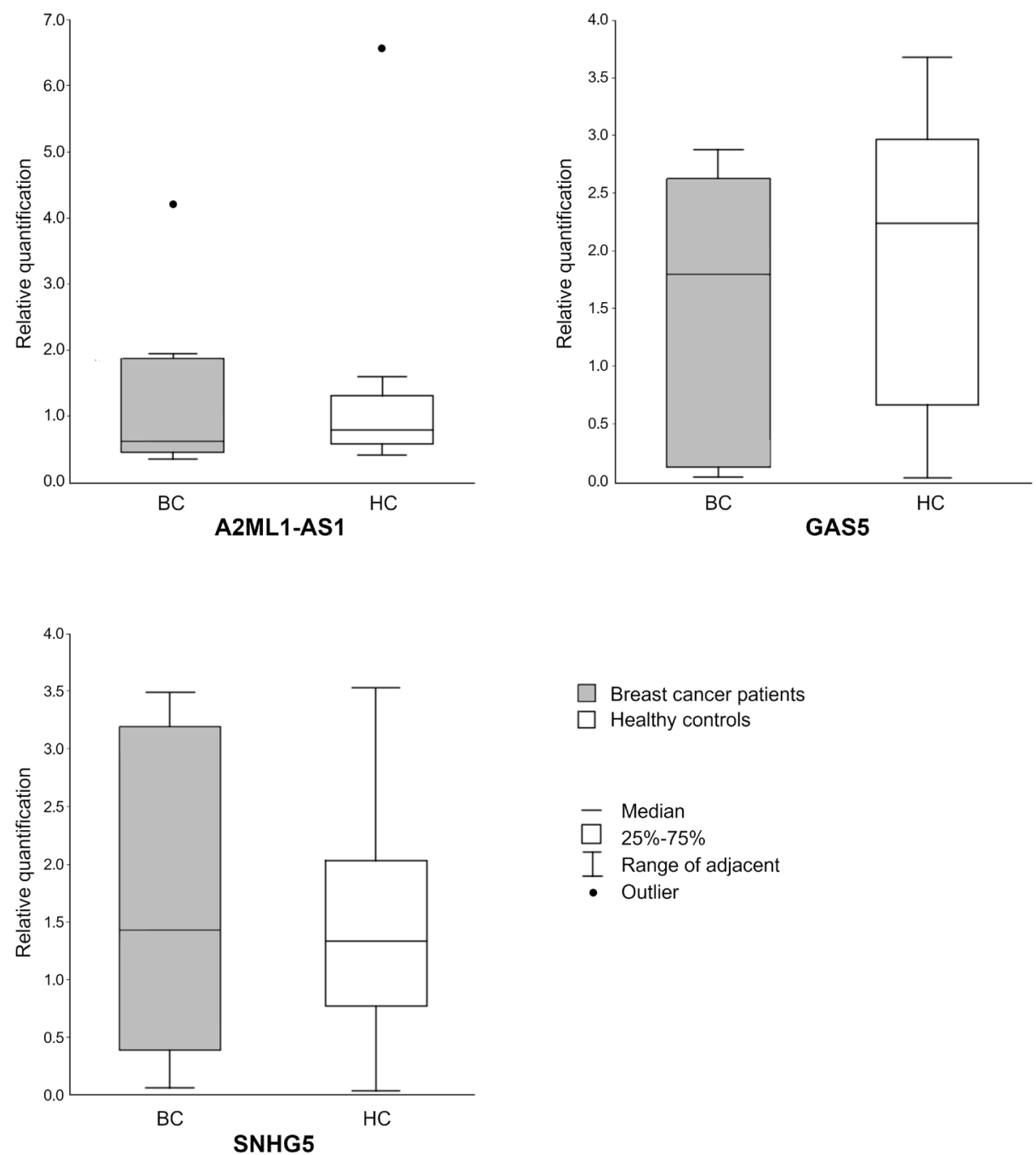

Breast cancer patients

$\square$ Healthy controls

- Median

$\square$ 25\%-75\%

I Range of adjacent

- Outlier

Figure 3. Relative quantification of most abundant lncRNAs in plasma of $\mathrm{BC}$ patients and healthy controls (Cq<35, normalization by global mean). 
in plasma samples of healthy individuals. Systemic examination of RNA quality performed in this study [25] revealed no evidence of RNA degradation or RT-qPCR inhibition. Using the same array technology, the analyzed lncRNAs were robustly detected in tissues [25]. Unfortunately, our study was not designed to compare the lncRNA levels in plasma samples and corresponding tissues. Schlosser et al. [25] examined a cohort of healthy volunteers but they failed to detect lncRNAs HOTAIR and MALAT I in plasma samples despite the carefully performed quality control steps. We detected HOTAIR in five out of the nine healthy subjects in lower concentrations than in the only one breast cancer patient. With regard to the performance of HOTAIR as a biomarker, there are inconclusive results: Zhang et al. [26] reported that the plasma levels of HOTAIR in ER-negative patients were lower than in ER-positive patients but GökmenPolar et al. [27] received opposite results in cancer tissues. It seems that the diagnostic success of HOTAIR examination will be highly dependent on the types of biological samples and on the abundance of particular BC subtypes in the analyzed datasets. In our pilot study, we were not able to find a characteristic, which would distinguish the patient no. 8 , with the very high level of HOTAIR in her plasma, from other patients (Table 1, Figure 3). Further large-scale studies are probably necessary for elucidation of these complex problems.

In opposite, GAS5 was detected by Schlosser et al. [25] in all examined plasma samples. We confirmed this result. Our results correspond also with the findings of a study where GAS5 was detected in plasma of all BC patients and healthy controls [16]. In agreement with this report [16], no difference in plasma GAS5 concentrations between BC patients and controls was found in our study (Figures 1 and 3 ). It has been repeatedly reported that the levels of HOTAIR were not stable in plasma $[16,25]$. We confirm this finding not only for HOTAIR but also for MALAT I (Figures 1).

Based on our results obtained with Human Inflammatory Response \& Autoimmunity $\mathrm{RT}^{2}$ lncRNA PCR Array, we can conclude that the lncRNAs previously proposed as potential markers for non-invasive breast cancer detection were not able to fulfill this function in this experimental setting on small randomly selected group of non-treated $\mathrm{BC}$ patients due to the detection of low or none concentrations in plasma samples (HOTAIR or MALAT I) or due to no differences between patients and controls (GAS5) (Figures 1, 2 and 3).

Santoro et al. [28] described a successful application of the identical methodology, which has been employed in our study for selection of potential biomarkers in serum of patients with multiple sclerosis.

In our hands, the methodology applied on plasma samples provided the information about the three most abundant lncRNAs which were present in all plasma samples independently on health status; GAS5 inhibits proliferation and stimulates apoptosis of different cell types. It acts as a tumor suppressor [29]. A2ML1 antisense RNA 1 contains tens of binding sites for different transcription factors (https://www. genecards.org) therefore it has probably an extreme regulatory potential. Small nucleolar RNA host gene 5 (SNHG5) is known to interact with 121 transcripts in cytoplasm and to block their degradation [30]. It was upregulated in triplenegative breast cancer cell lines [31].

According to the results published by Schlosser et al. [25], we can conclude that the examined technological platform is able to detect the most abundant and stable lncRNAs in plasma. Actually, there is still a lack of references describing the types of lncRNAs together with their relative abundance in plasma of healthy individuals. In this context, it is also important to stress the fact that there is no reliable method for the quality control of RNA species isolated from plasma samples, as RIN values cannot be used [23] as discussed in Material and Methods. Our results provide the information about some of the most frequently occurring lncRNA in plasma and represent the limited contribution to the understanding of the complex problem. The Next Generation Sequencing technology is very promising with regard to the lncRNA profiling in biological samples but also the results of such an advanced technology should to be validated by an independent technological platform - mostly by single target RT-quantitative PCR - to provide reliable information.

Acknowledgements: This work was supported by the Ministry of Education, Youth and Sport of the Czech Republic (grant numbers Progres Q25 and SVV 260 373) and by the Ministry of Health of the Czech Republic (grant number RVO/VFN 64165).

\section{References}

[1] DUFFY MJ, EVOY D, MCDERMOTT EW. CA 15-3: uses and limitation as a biomarker for breast cancer. Clin Chim Acta 2010; 411: 1869-1874. https://doi.org/10.1016/j. cca.2010.08.039

[2] SCHWARZENBACH H, HOON DS, PANTEL K. Cell-free nucleic acids as biomarkers in cancer patients. Nat Rev Cancer 2011; 11: 426-437. https://doi.org/10.1038/nrc3066

[3] QIN Z, LJUBIMOV VA, ZHOU C, TONG Y, LIANG J. Cellfree circulating tumor DNA in cancer. Chin J Cancer 2016; 35: 36. https://doi.org/10.1186/s40880-016-0092-4

[4] ALIX-PANABIERES C, PANTEL K. Clinical Applications of Circulating Tumor Cells and Circulating Tumor DNA as Liquid Biopsy. Cancer Discov 2016; 6: 479-491. https://doi. org/10.1158/2159-8290.CD-15-1483

[5] JARRY J, SCHADENDORF D, GREENWOOD C, SPATZ A, VAN KEMPEN LC. The validity of circulating microRNAs in oncology: five years of challenges and contradictions. Mol Oncol 2014; 8: 819-829. https://doi.org/10.1016/j. molonc.2014.02.009

[6] TANTAI J, HU D, YANG Y, GENG J. Combined identification of long non-coding RNA XIST and HIF1A-AS1 in serum as an effective screening for non-small cell lung cancer. Int J Clin Exp Pathol 2015; 8: 7887-7895. 
[7] CREA F, WATAHIKI A, QUAGLIATA L, XUE H, PIKOR $\mathrm{L}$ et al. Identification of a long non-coding RNA as a novel biomarker and potential therapeutic target for metastatic prostate cancer. Oncotarget 2014; 5: 764-774. https://doi. org/10.18632/oncotarget.1769

[8] GUO X, GAO L, WANG Y, CHIU DK, WANG T et al. Advances in long noncoding RNAs: identification, structure prediction and function annotation. Brief Funct Genomics 2016; 15: 38-46. https://doi.org/10.1093/bfgp/elv022

[9] REDON S, REICHENBACH P, LINGNER J. The non-coding RNA TERRA is a natural ligand and direct inhibitor of human telomerase. Nucleic Acids Res 2010; 38: 5797-5806. https://doi.org/10.1093/nar/gkq296

[10] RINN JL, KERTESZ M, WANG JK, SQUAZZO SL, XU X et al. Functional demarcation of active and silent chromatin domains in human HOX loci by noncoding RNAs. Cell 2007; 129: 1311-1323. https://doi.org/10.1016/j.cell.2007.05.022

[11] FROBERG JE, YANG L, LEE JT. Guided by RNAs: X-inactivation as a model for lncRNA function. J Mol Biol 2013; 425: 3698-3706. https://doi.org/10.1016/j.jmb.2013.06.031

[12] WANG K, LIU F, ZHOU LY, LONG B, YUAN SM et al. The long noncoding RNA CHRF regulates cardiac hypertrophy by targeting miR-489. Circ Res 2014; 114: 1377-1388. https://doi.org/10.1161/CIRCRESAHA.114.302476

[13] ZHANG H, ZEITZ MJ, WANG H, NIU B, GE S, et al. Long noncoding RNA-mediated intrachromosomal interactions promote imprinting at the Kcnq1 locus. J Cell Biol 2014; 204: 61-75. https://doi.org/10.1083/jcb.201304152

[14] CHEN WM, HUANG MD, KONG R, XU TP, ZHANG EB et al. Antisense Long Noncoding RNA HIF1A-AS2 Is Upregulated in Gastric Cancer and Associated with Poor Prognosis. Dig Dis Sci 2015; 60: 1655-1662. https://doi.org/10.1007/ s10620-015-3524-0

[15] LI W, ZHAI L, WANG H, LIU C, ZHANG J et al. Downregulation of LncRNA GAS5 causes trastuzumab resistance in breast cancer. Oncotarget 2016; 7: 27778-27786. https:// doi.org/10.18632/oncotarget.8413

[16] HAN L, MA P, LIU SM, ZHOU X. Circulating long noncoding RNA GAS5 as a potential biomarker in breast cancer for assessing the surgical effects. Tumour Biol 2015; 37: 68476854. https://doi.org/10.1007/s13277-015-4568-7

[17] YU G, ZHANG W, ZHU L, XIA L. Upregulated long noncoding RNAs demonstrate promising efficacy for breast cancer detection: a meta-analysis. Onco Targets Ther 2018; 11: 1491-1499. https://doi.org/10.2147/OTT.S152241

[18] WHITING PF, RUTJES AW, WESTWOOD ME, MALLETT S, DEEKS JJ et al. QUADAS-2: a revised tool for the quality assessment of diagnostic accuracy studies. Ann Intern Med 2011; 155: 529-536. https://doi.org/10.7326/0003-4819-1558-201110180-00009

[19] BHAN A, MANDAL SS. LncRNA HOTAIR: A master regulator of chromatin dynamics and cancer. Biochim Biophys Acta 2015; 1856: 151-164. https://doi.org/10.1016/j. bbcan.2015.07.001
[20] SOUDYAB M, IRANPOUR M, GHAFOURI-FARD S. The Role of Long Non-Coding RNAs in Breast Cancer. Arch Iran Med 2016; 19: 508-517. https://doi.org/0161907/ AIM.0011

[21] FITZGERALD KA, CAFFREY DR. Long noncoding RNAs in innate and adaptive immunity. Curr Opin Immunol 2014; 26: 140-146. https://doi.org/10.1016/j.coi.2013.12.001

[22] YU WD, WANG H, HE QF, XU Y, WANG XC. Long noncoding RNAs in cancer-immunity cycle. J Cell Physiol 2018; 233: 6518-6523. https://doi.org/10.1002/jcp.26568

[23] HILL AF, PEGTEL DM, LAMBERTZ U, LEONARDI T, O'DRISCOLL L et al. ISEV position paper: extracellular vesicle RNA analysis and bioinformatics. J Extracell Vesicles 2013; 2. https://doi.org/10.3402/jev.v2i0.22859

[24] SUN Z, YANG S, ZHOU Q, WANG G, SONG J et al. Emerging role of exosome-derived long non-coding RNAs in tumor microenvironment. Mol Cancer 2018; 17: 82. https:// doi.org/10.1186/s12943-018-0831-Z

[25] SCHLOSSER K, HANSON J, VILLENEUVE PJ, DIMITROULAKOS J, MCINTYRE L et al. Assessment of Circulating LncRNAs Under Physiologic and Pathologic Conditions in Humans Reveals Potential Limitations as Biomarkers. Sci Rep 2016; 6: 36596. https://doi.org/10.1038/ srep36596

[26] ZHANG Y, ZHANG K, LUO Z, LIU L, WU L et al. Circulating long non-coding HOX transcript antisense intergenic ribonucleic acid in plasma as a potential biomarker for diagnosis of breast cancer. Thorac Cancer 2016; 7: 627-632. https://doi.org/10.1111/1759-7714.12373

[27] GOKMEN-POLAR Y, VLADISLAV IT, NEELAMRAJU Y, JANGA SC, BADVE S. Prognostic impact of HOTAIR expression is restricted to ER-negative breast cancers. Sci Rep 2015; 5: 8765. https://doi.org/10.1038/srep08765

[28] SANTORO M, NOCITI V, LUCCHINI M, DE FINO C, LOSAVIO FA et al. Expression Profile of Long Non-Coding RNAs in Serum of Patients with Multiple Sclerosis. J Mol Neurosci 2016; 59: 18-23. https://doi.org/10.1007/s12031016-0741-8

[29] PICKARD MR, WILLIAMS GT. Molecular and Cellular Mechanisms of Action of Tumour Suppressor GAS5 LncRNA. Genes (Basel) 2015; 6: 484-499. https://doi. org/10.3390/genes6030484

[30] DAMAS ND, MARCATTI M, COME C, CHRISTENSEN LL, NIELSEN MM et al. SNHG5 promotes colorectal cancer cell survival by counteracting STAU1-mediated mRNA destabilization. Nat Commun 2016; 7: 13875. https://doi. org/10.1038/ncomms 13875

[31] LEE J, JUNG JH, CHAE YS, PARK HY, KIM WW et al. Long Noncoding RNA snaR Regulates Proliferation, Migration and Invasion of Triple-negative Breast Cancer Cells. Anticancer Res 2016; 36: 6289-6295. https://doi.org/10.21873/ anticanres.11224 